\title{
VmeAB, an RND-type multidrug efflux transporter in Vibrio parahaemolyticus
}

\author{
Correspondence \\ Teruo Kuroda \\ tkuroda@cc.okayama-u.ac.jp
}

Received 9 May 2007

Revised 6 August 2007

Accepted 13 September 2007

\author{
Taira Matsuo, ${ }^{1} \dagger$ Katsuhiko Hayashi, ${ }^{2} \dagger$ Yuji Morita, ${ }^{2} \ddagger$ \\ Motohiro Koterasawa, ${ }^{2}$ Wakano Ogawa, ${ }^{2}$ Tohru Mizushima, ${ }^{2} \S$ \\ Tomofusa Tsuchiya $^{2}$ and Teruo Kuroda ${ }^{1}$ \\ ${ }^{1}$ Department of Genome Applied Microbiology, Graduate School of Medicine, Dentistry and \\ Pharmaceutical Sciences, Okayama University, Tsushima, Okayama 700-8530, Japan \\ ${ }^{2}$ Department of Molecular Microbiology, Graduate School of Medicine, Dentistry and \\ Pharmaceutical Sciences, Okayama University, Tsushima, Okayama 700-8530, Japan
}

Genes $v m e A$ and $v m e B$, encoding a multidrug efflux transporter in the halophilic bacterium Vibrio parahaemolyticus, have been cloned using a drug-hypersusceptible Escherichia coli strain as the host. Cells of $E$. coli KAM33 ( $\triangle a c r A B \Delta y d h E)$ carrying the vmeAB region from $V$.

parahaemolyticus conferred much higher MICs for a variety of antimicrobial agents than did control cells. Cells possessing VmeAB under energized conditions maintained very low intracellular concentrations of ethidium. This was as expected for an energy-dependent efflux system, and supports the notion - based on sequence homology - that VmeAB belongs to the resistance nodulation cell division (RND) family of multidrug efflux transporters. It is likely that VmeAB forms functional complexes with the outer-membrane protein TolC in $E$. coli, because introduction of $v m e A B$ into cells of $E$. coli KAM43, which lacks the to/C gene, failed to elevate the MICs for any of the antimicrobial agents tested. Therefore, a $V$. parahaemolyticus homologue of to/C was also cloned, designated $v p o C$, and was introduced together with vme $A B$ into cells of $E$. coli KAM43. The MICs of all agents tested were raised and were comparable to the values observed in $E$. coli KАMЗ3 harbouring a plasmid carrying vmeAB. Finally, a $v m e A B$-deficient mutant of $V$. parahaemolyticus was constructed (designated TM3). TM3 showed slightly higher susceptibility than the parental $V$. parahaemolyticus to some antimicrobial agents. Survival rate of the TM3 when exposed to deoxycholate decreased compared with that of the parent.

\section{INTRODUCTION}

Vibrio parahaemolyticus, a slightly halophilic marine bacterium, is a major cause of food poisoning in Japan (Obata et al., 2001; WHO, 1999; Yamazaki et al., 2003) and many other countries (Cabanillas-Beltran et al., 2006; CDC, 2006; Lozano-Leon et al., 2003; McLaughlin et al., 2005; Sen et al., 2007; Su et al., 2005a). The organism has some peculiar physiological characteristics, such as a very fast growth rate, $\mathrm{a} \mathrm{Na}^{+}$requirement for growth (Baumann \& Schubert, 1984) and an ability to live in diverse

†These authors contributed equally to this work.

$\ddagger$ Present address: Department of Microbiology, School of Pharmacy, Aichi Gakuin University, Nagoya 464-8650, Japan.

§Present address: Graduate School of Medical and Pharmaceutical Sciences. Kumamoto University, Kumamoto 862-0973, Japan.

Abbreviations: FLP, flippase; FRT, FLP recombinase recognition target; MATE, multidrug and toxic compound extrusion; RND, resistance nodulation cell division; TPP-Cl, tetraphenylphosphonium chloride.

The GenBank/EMBL/DDBJ accession number for the sequence reported in this paper is AB251606 (vmeAB). environments including brackish water (Kumazawa \& Kato, 1985), on estuarine algae (Kumazawa et al., 1991), and in mammalian hosts, including humans (Yamamoto \& Yokota, 1989).

In achieving human infection, $V$. parahaemolyticus survives in the intestine and especially in the duodenum, where bile acids and their conjugates are abundant. Bile acids are anionic detergents and support the digestion of fats in the intestine. In addition, bile has bactericidal effects due to its membrane-solvent property (Provenzano et al., 2000). Thus, bile resistance of enteropathogenic bacteria is thought to be important for their survival in the intestine.

Some enteropathogenic bacteria have been shown to possess systems to protect from the actions of bile. In the case of Vibrio cholerae, the outer-membrane component $\mathrm{TolC}_{\mathrm{Vc}}$, which had already been identified as a component of some multidrug efflux transporters (sometimes called multidrug efflux pumps), was found to contribute to resistance to bile, cholate and deoxycholate (Bina \& Mekalanos, 2001). In the case of Escherichia coli, energydependent efflux of bile salts was observed (Thanassi et al., 
1997). Moreover, several multidrug efflux transporters have already been described as having involvement in resistance to deoxycholate and other constituents of bile (Nishino \& Yamaguchi, 2001; Nishino et al., 2003; Xu et al., 2003), thus implying that the multidrug efflux transporters themselves play a significant role in resistance to bile, as well as to other antimicrobial agents.

Recently, numerous multidrug efflux transporters have been characterized in micro-organisms. These proteins can be classified into five families, according to their primary structure and energy source for transport. These are (i) RND (resistance nodulation cell division), (ii) MF (major facilitator), (iii) MATE (multidrug and toxic compound extrusion), (iv) SMR (small multidrug resistance) and (v) ABC (ATP-binding cassette) families (Piddock, 2006). From the available databases of genome sequences (Makino et al., 2003), we estimated that there were about 50 multidrug efflux transporters in V. parahaemolyticus, according to their primary structure similarities. Therefore, we have set out to clone the multidrug transporter genes from $V$. parahaemolyticus, and to characterize the properties of the transporters.

In previous papers, we have reported on the cloning and properties of two transporters in the MATE family, NorM and VmrA from $V$. parahaemolyticus (Chen et al., 2002; Morita et al., 1998, 2000). Here, we describe cloning of the $v m e A$ and $v m e B$ genes in E. coli KAM3, and characterization of the encoded multidrug efflux transporter $V m e A B$, which belongs to the RND family. Cells possessing VmeAB conferred much higher MICs than did control cells, of a variety of antimicrobial agents. Moreover, vmeAB-deficient $V$. parahaemolyticus showed a reduced survival rate in the presence of deoxycholate. This is believed to be the first description of an RND-type transporter in $V$. parahaemolyticus.

\section{METHODS}

Bacterial strains and growth. V. parahaemolyticus AQ3334 (Chen et al., 2002; Kuroda et al., 2005; Morita et al., 1998) was used as the source of chromosomal DNA. E. coli KAM3 ( $\triangle a c r B)$ (Masaoka et al., 2000; Mine et al., 1999), KAM33 ( $\triangle a c r A B \Delta y d h E)$ and KAM43 $(\triangle a c r A B \triangle y d h E \Delta t o l C)$ were used as hosts for gene cloning and transporter characterization. The constructions of KAM33 and KAM43 are described below. E. coli TG1 $\Delta$ tolC was provided by A. Yamaguchi, Osaka University, Japan, and E. coli $\beta 2155$ was provided by D. Mazel, Institut Pasteur, France. V. parahaemolyticus was grown in LB medium ( $1 \%$ polypeptone, $0.5 \%$ yeast extract, $1 \% \mathrm{NaCl}$, $\mathrm{pH} 7.0$ ), and E. coli was grown in L medium (1\% polypeptone, $0.5 \%$ yeast extract, $0.5 \% \mathrm{NaCl}, \mathrm{pH} 7.0)$. Cell growth was monitored by determining the turbidity at $650 \mathrm{~nm}$.

Construction of $\boldsymbol{E}$. coli KAM33 and KAM43. E. coli KAM33 was constructed by using the gene-deletion method of Datsenko \& Wanner (2000). Plasmids (pKD3, pKD46, pCP20) were kindly provided by B. L. Wanner, Purdue University, USA. PCR products were generated by using primers described below. The primers for $a c r A B$ disruption were acrANFRT (5'-atgaacaaaaacagagggtttacgcctctggcggtcagggatgtaacgcactgagaag- $\left.3^{\prime}\right)$ and acrBCFRT $\left(5^{\prime}-\right.$ tcaatgatgatcgacagtatggctgtgctcgatatcacgtcttgagcgattgtgtagg- $\left.3^{\prime}\right)$. These primers included homology extensions of those genes and priming sequences for pKD3 as template. KAM32 ( $\triangle a c r B \Delta y d h E)$ (Chen et al., 2002) was used as a parent for the construction of KAM33, and the resulting KAM33 was then used as the parent for construction of KAM43. In the case of the construction of KAM33, E. coli KAM32 cells carrying a Red helper plasmid (pKD46) were grown in $5 \mathrm{ml} \mathrm{SOB}$ ( $2 \%$ Bacto Tryptone, $0.5 \%$ yeast extract, $10 \mathrm{mM} \mathrm{NaCl}, 2.5 \mathrm{mM} \mathrm{KCl}$, $10 \mathrm{mM} \mathrm{MgCl}_{2}$ ) containing $100 \mu \mathrm{g}$ ampicillin $\mathrm{ml}^{-1}$ and $0.2 \% \mathrm{~L}^{-}$ arabinose at $30{ }^{\circ} \mathrm{C}$ until mid-exponential phase. Harvested cells were washed three times with ice-cold distilled water and concentrated 100 -fold. PCR products were gel-purified and suspended in distilled water. Electroporation was done by using a Gene-pulser (Bio-Rad) according to the manufacturer's instructions, with $50 \mu$ concentrated cells and $10 \mathrm{ng}$ PCR product. One millilitre of pre-warmed SOC ( $2 \%$ Bacto Tryptone, $0.5 \%$ yeast extract, $10 \mathrm{mM} \mathrm{NaCl}, 2.5 \mathrm{mM} \mathrm{KCl}$, $10 \mathrm{mM} \mathrm{MgCl}, 0.1 \%$ glucose) containing $0.1 \%$ L-arabinose was added to the shocked cells, and incubated for $2 \mathrm{~h}$ at $37^{\circ} \mathrm{C}$. Cells were spread onto agar and incubated at $30{ }^{\circ} \mathrm{C}$ to select the chloramphenicol-resistant $\left(\mathrm{Cm}^{\mathrm{R}}\right)$ transformants. These candidates were streaked on medium without an antibiotic and incubated at $42{ }^{\circ} \mathrm{C} . \mathrm{Cm}^{\mathrm{R}}$ and ampicillin-sensitive candidates were selected for a further step.

The $\mathrm{Cm}^{\mathrm{R}}$ gene can be eliminated by using a helper plasmid expressing the flippase (FLP) recombinase, which acts on the directly repeated FRT (FLP recognition target) sites flanking the resistance gene (Cherepanov \& Wackernagel, 1995; Datsenko \& Wanner, 2000). $\mathrm{Cm}^{\mathrm{R}}$ candidates were transformed with pCP20, which carried the FLP recombinase gene, and ampicillin-resistant transformants were selected at $30{ }^{\circ} \mathrm{C}$. After non-selective single-colony isolation at $42{ }^{\circ} \mathrm{C}$ for $24 \mathrm{~h}$, the isolates were then tested for loss of chloramphenicol and ampicillin resistances. The majority had lost the FRT-flanked resistance gene and the FLP helper plasmid simultaneously. The disruption of genes was confirmed by PCR analysis.

Prior to construction of KAM43 ( $\triangle a c r A B \quad \Delta y d h E \Delta t o l C)$, we constructed KAM42 ( $\Delta a c r B \Delta y d h E \Delta t o l C)$ from KAM32 ( $\triangle a c r B$ $\Delta y d h E$ ) (Chen et al., 2002). For disruption of tolC, PCR products were amplified with two primers, tolCFRTN2 (5'-gatcgcgctaaatactgcttcaccacaaggaatgcaaatggtgtaggctggagctgcttc- $3^{\prime}$ ) and tolCFRTC2 ( $5^{\prime}$-gtcatcagttacggaaagggttatgaccgttactggtggtcatgggaattagccatggtc- $\left.3^{\prime}\right)$, using pKD3 as the template. The PCR products were introduced by electroporation into the parental KAM32 carrying pKD46. $\mathrm{Cm}^{\mathrm{R}}$ candidates were selected, and insertion of the $\mathrm{Cm}^{\mathrm{R}}$ gene in the tolC was confirmed by the PCR method. A region of tolC:: $\mathrm{Cm}^{\mathrm{R}}$ was transferred into KAM33 by P1 transduction, and then elimination of FRT-flanked resistance gene was carried out as described above.

Gene cloning and sequencing. Chromosomal DNA was prepared from the $V$. parahaemolyticus cells by the method of Berns \& Thomas (1965). The DNA was partially digested with Sau3AI, and 4-10 kb fragments were separated by sucrose density-gradient centrifugation. Vector plasmid pBR322 was digested with BamHI, dephosphorylated with bacterial alkaline phosphatase, and then ligated with the chromosomal DNA fragments by using ligation kit version 2 (TaKaRa BIO). Competent cells of E. coli KAM3, prepared by the method of Hanahan et al. (1991), were transformed with the recombinant plasmids. The transformed cells were spread onto agar plates containing $\mathrm{L}$ medium, $10 \mu \mathrm{g}$ rhodamine $6 \mathrm{G} \mathrm{ml} \mathrm{m}^{-1}, 100 \mu \mathrm{g}$ ampicillin $\mathrm{ml}^{-1}$ and $1.5 \%$ agar, on which E. coli KAM3 could not grow. The plates were incubated at $37{ }^{\circ} \mathrm{C}$ for $48 \mathrm{~h}$. Candidate colonies that appeared on the plate were purified by single-colony isolation, and growth on the plate containing the same drugs as described above was checked. Plasmids were isolated from each of the candidates. Those candidate plasmids were reintroduced into KAM3 cells, and the cells were spread onto an identical plate. Plasmids were isolated from each of these transformants. One of the candidate plasmids was named pRHR2. 
To assess the contribution of the cloned resistance determinant to $\beta$ lactams, we also constructed pRHR229, in which the NsiI-SphI fragment of pRHR2 was inserted into the multicloning site of pSTV29 (TaKaRa BIO). Plasmid pSTV29 carries the $\mathrm{Cm}^{\mathrm{R}}$ gene, but not the $\beta$ lactamase gene. Plasmid pRHR229 had the shortest insert, and transformed cells harbouring this plasmid were resistant to several antimicrobial agents. For sequencing, plasmid pRHR222, which carried the PvuII-SalI fragment of pRHR2 in pBluescript SK $(+)$ (Stratagene), was constructed. Deletion plasmids for sequencing were constructed using exonuclease III and mung bean nuclease (Henikoff, 1984).

Cloning of vpoC (VP0425). Cloning of VP0425 was carried out by PCR. Template DNA was prepared as described above, from $V$. parahaemolyticus RIMD2210633, for which the genome sequence is available (http://genome.naist.jp/bacteria/vpara/). The two primers were VP0425-F (5'-tactggatccgcgggaatagaaaatgaa-3') and VP0425-R ( $5^{\prime}$-cgagggatccgcgttttaatttcattgta- $\left.3^{\prime}\right)$; the PCR product was ligated into pBR322 at the BamHI site. To facilitate the cloning, a BamHI restriction site was introduced at the $5^{\prime}$ end of both primers. The resulting plasmid was designated pBVT3.

Drug susceptibility test. The MICs of various antimicrobial agents were determined by the microdilution method according to the recommendations of the Japanese Society of Chemotherapy (1990). Briefly, MICs were determined in Mueller-Hinton broth (Difco) containing each compound in a twofold serial dilution series. The cells were incubated in the test medium at $37{ }^{\circ} \mathrm{C}$ for $24 \mathrm{~h}$, and growth was examined visually. The MIC of each compound was defined as the lowest concentration that prevented visible growth.

Ethidium accumulation assay. Ethidium accumulation assays were performed as described previously (Xu et al., 2003).

Disruption of vmeAB in V. parahaemolyticus. The plasmid pRHR228 was constructed using the same procedure as for pRHR229 (described above), but using pSTV28 (TaKaRa BIO) instead of pSTV29. It also carries the whole $v m e A B$ region. There are two $H p a I$ sites in the $v m e A B$ region, one in $v m e A$ and another in $v m e B$. Plasmid pRHR228 was digested with $H p a I$, and the $H p a I$ fragment covering a large portion of $v m e A B$ was removed by self-ligation. A SalI-XbaI fragment carrying the incomplete $v m e A B$ region lacking the Hpal fragment was cut out and ligated to the suicide vector pXAC623 (Kuroda et al., 2005), which had been digested with SalI and XbaI. The resulting plasmid, pXAC1092, carries the incomplete $v m e A B$, the cat gene and the $s a c B$ gene.

Plasmid pXAC1092 was introduced into cells of E. coli donor strain $\beta 2155$ (Herz et al., 2003). Mating between donor cells of E. coli $\beta 2155 /$ pXAC1092 and recipient cells of $V$. parahaemolyticus AQ3334 was carried out as described previously (Kuroda et al., 2005). Briefly, donor cells were grown in LB medium supplemented with $0.5 \mathrm{mM}$ diaminopimelic acid and $20 \mu \mathrm{g}$ chloramphenicol $\mathrm{ml}^{-1}$, and recipient cells were grown in LB medium. Both cells were harvested at the early exponential phase of growth, mixed gently, and trapped on a membrane filter $(0.2 \mu \mathrm{m}$ pore size, ADVANTEC Toyo). After washing, the filter was put on an LB medium agar plate (1.5\% agar) and incubated at $37{ }^{\circ} \mathrm{C}$ for $3 \mathrm{~h}$. The cells were suspended in $\mathrm{LB}$ medium, and shaken at $37{ }^{\circ} \mathrm{C}$ for $1 \mathrm{~h}$. Candidate colonies of $\mathrm{V}$. parahaemolyticus, into the genome of which pXAC1092 had been integrated, could be obtained on agar plates containing $5 \mu \mathrm{g}$ chloramphenicol $\mathrm{ml}^{-1}$ and $100 \mu \mathrm{g}$ ampicillin $\mathrm{ml}^{-1}$, since $V$. parahaemolyticus AQ3334 is resistant to ampicillin at this concentration but $E$. coli $\beta 2155$ is susceptible. The candidates were cultured in LB medium without antibiotics, spread on VDS-broth agar plates ( $1 \%$ polypeptone, $0.5 \%$ yeast extract, $30 \mathrm{mM} \mathrm{NaCl}, 55 \mathrm{mM} \mathrm{KCl}$, $10 \%$ sucrose, $\mathrm{pH} 7.0,2.5 \%$ agar) and incubated at $25{ }^{\circ} \mathrm{C}$. Occurrence of a second recombination should yield cells resistant to sucrose but susceptible to chloramphenicol, and several such candidates were identified. After checking deletion of the $v m e A B$ region by PCR, we picked a single isolate for further use and designated it TM3.

Transformation of $V$. parahaemolyticus was carried out by the method of Hamashima et al. (1995). V. parahaemolyticus cells were cultured in LB medium until mid-exponential phase was reached. Harvested cells were washed with $20 \mathrm{ml}$ ice-cold EP buffer $(272 \mathrm{mM}$ sucrose, $1 \mathrm{mM}$ $\left.\mathrm{MgCl}_{2}, 7 \mathrm{mM} \mathrm{KH_{2 }} \mathrm{PO}_{4} / \mathrm{Na}_{2} \mathrm{HPO}_{4}, \mathrm{pH} 7.4\right)$ and the pellet was resuspended in $2 \mathrm{ml}$ of the same buffer. One microgram of pSTV29 or pRHR229 was added into $200 \mu \mathrm{l}$ of the cell suspension. The mixture was placed on ice for $15 \mathrm{~min}$ and transferred to a chilled cuvette with a distance of $0.2 \mathrm{~cm}$ between electrodes, and pulsed with a time constant of $25 \mathrm{~ms}(25 \mu \mathrm{F}$ capacitance, $1000 \Omega)$ at $1.4 \mathrm{kV}$ by using a Gene-pulser (Bio-Rad) according to the manufacturer's instructions. Shocked cells were added into $800 \mu \mathrm{l}$ pre-warmed LB medium, and incubated at $37{ }^{\circ} \mathrm{C}$ for $1 \mathrm{~h}$. Transformants were selected on an LB plate containing $10 \mu \mathrm{g}$ chloramphenicol ml $\mathrm{m}^{-1}$ (2.5\% agar).

Effect of sodium deoxycholate on survival rate of $\boldsymbol{V}$. parahaemolyticus strains. Approximately $2 \times 10^{8}$ cells of $V$. parahaemolyticus were suspended in $1 \mathrm{ml}$ of $0.5 \mathrm{M} \mathrm{NaCl}-\mathrm{PB}(0.1 \mathrm{M}$ phosphate buffer, $\mathrm{pH} 7.2$ ) including $1 \mathrm{mg}$ sodium deoxycholate $\mathrm{ml}^{-1}$. After incubation at $37^{\circ} \mathrm{C}$ for $10 \mathrm{~min}$, cells diluted $10^{-4}$ with $0.5 \mathrm{M} \mathrm{NaCl}-\mathrm{PB}$ were spread onto an LB agar plate (2.5\% agar). Following incubation at $37{ }^{\circ} \mathrm{C}$ for $12 \mathrm{~h}$, c.f.u. were counted.

\section{RESULTS}

\section{Cloning of multidrug resistance gene}

Using E. coli KAM3 as the host, we cloned a DNA fragment that enabled KAM3 cells to grow in the presence of $10 \mu \mathrm{g}$ rhodamine $6 \mathrm{G} \mathrm{ml}^{-1}$. E. coli KAM3 could not grow on this plate. After incubation at $37{ }^{\circ} \mathrm{C}$ for $48 \mathrm{~h}$, some candidate colonies appeared. Plasmids isolated from the candidates were reintroduced into KAM3 cells, and the cells could grow on the same medium. Since rhodamine $6 \mathrm{G}$ was shown to be a good substrate for some multidrug transporters (Chen et al., 2003; Huda et al., 2003; Nishino \& Yamaguchi, 2001; Sekiya et al., 2003; Su et al., 2005b), multidrug transporter genes were suggested to be included in these candidate plasmids. One of the candidate plasmids was named pRHR2.

\section{Sequencing}

Upon sequencing of the plasmid insert that conferred elevated MICs of several antimicrobial agents, we found two ORFs. Both of these were required for the elevated MICs and we designated them vmeA and vmeB. Comparison of the new sequences with the published genome sequence of $V$. parahaemolyticus (Makino et al., 2003) showed vmeA to correspond to VP1091, and vmeB to $V P 1092$. Fourteen differences of nucleotide sequence were identified between vmeA and VP1091, and 37 differences between $v m e B$ and VP1092. All these differences were translationally silent, except two: changing amino acid residue Gly33 in VP1091 to Ala33 in VmeA, and changing Ala1046 in VP1092 to Asp1046 in VmeB. A BLAST search 
for protein sequence similarities (NCBI) showed VmeA to share, respectively, 49, 49, 47 and $46 \%$ identities with AcrA and AcrE of E. coli (Klein et al., 1991; Ma et al., 1993), SdeX of Serratia marcescens (Chen et al., 2003) and MexA of Pseudomonas aeruginosa (Poole et al., 1993). All of these are so-called membrane fusion proteins (MFPs) of RNDtype multidrug efflux transporters. The analysis also showed VmeB to share $63,63,62,62$ and $61 \%$ identities, respectively, with AcrB, AcrD and AcrF of E. coli (Klein et al., 1991; Ma et al., 1993, 1994), SdeY of S. marcescens (Chen et al., 2003) and MexB of P. aeruginosa (Poole et al., 1993). These are inner-membrane proteins of RND-type multidrug efflux transporters. Thus, VmeAB seems to be a solid member of the RND family of multidrug efflux transporters. Multidrug efflux transporters of the RND family have been shown to play an important role in intrinsic resistance to various antimicrobial agents in various Gram-negative bacteria, such as $E$. coli and $P$. aeruginosa (Li et al., 1995; Morita et al., 2001; Okusu et al., 1996; Poole et al., 1993; Tseng et al., 1999). We then further analysed VmeAB.

We found no ORF in the downstream region of $v m e B$ that might code for an outer-membrane component of this RND-type efflux transporter.

\section{Substrate specificity of VmeAB}

To characterize VmeAB, we determined the MICs of various antimicrobial agents using E. coli strain KAM33, made to be drug-hypersusceptible by deletion of the major multidrug efflux transporter, AcrAB. After plasmid pRHR229, carrying vmeAB, was introduced into KAM33, the resulting strain KAM33/pRHR229 displayed elevated MICs of rhodamine 6G, cloxacillin, oxacillin, erythromycin, tetracycline, norfloxacin, novobiocin, trimethoprim, benzalkonium chloride, acriflavine, crystal violet, ethidium bromide, sodium deoxycholate, SDS and tetraphenylphosphonium chloride (TPP-Cl), as shown in Table 1, compared with the control (E. coli KAM33/pSTV29). Since plasmid pSTV29 carries the $\mathrm{Cm}^{\mathrm{R}}$ gene, we also investigated the MIC of chloramphenicol for KAM33/ pRHR2. In addition to the antimicrobial agents described above, KAM33/pRHR2 showed four times higher MICs of chloramphenicol than KAM33/pBR322 (data not shown). Clearly, VmeAB contributes to multidrug resistance, when expressed in cells of $E$. coli, and its resistance spectrum is very similar to that of the AcrAB transporter in E. coli (Nishino \& Yamaguchi, 2001). There was no change in the MICs of aztreonam, carbenicillin, cefotaxime, imipenem, kanamycin or chlorhexidine gluconate (data not shown).

\section{Ethidium extrusion via VmeAB}

In order to demonstrate the transport activity of $\mathrm{VmeAB}$ directly, we measured intracellular ethidium levels of $E$. coli KAM33/pRHR229 exposed to $40 \mu \mathrm{M}$ ethidium bromide. Accumulation of ethidium in cells of KAM33/pRHR229
Table 1. MICs of various antimicrobial agents for $E$. coli KAM33/pRHR229

\begin{tabular}{|c|c|c|}
\hline \multirow[t]{2}{*}{ Antimicrobial agent } & \multicolumn{2}{|c|}{$\operatorname{MIC}\left(\mu \mathrm{g} \mathrm{ml}^{-1}\right)$} \\
\hline & $\begin{array}{c}\text { KAM33/pSTV29 } \\
\text { (control) }\end{array}$ & $\begin{array}{c}\text { KAM33/pRHR229 } \\
(\text { vmeAB })\end{array}$ \\
\hline Rhodamine $6 \mathrm{G}$ & 8 & 128 \\
\hline Cloxacillin & 1 & 64 \\
\hline Oxacillin & 0.5 & 128 \\
\hline Erythromycin & 4 & 16 \\
\hline Tetracycline & 0.5 & 1 \\
\hline Norfloxacin & 0.03 & 0.13 \\
\hline Novobiocin & 2 & 128 \\
\hline Trimethoprim & 0.06 & 0.13 \\
\hline Benzalkonium chloride & 4 & 16 \\
\hline Acriflavine & 2 & 32 \\
\hline Crystal violet & 4 & 16 \\
\hline Ethidium bromide & 4 & 256 \\
\hline Sodium deoxycholate & 1000 & $>32000$ \\
\hline SDS & 100 & $>51200$ \\
\hline TPP-Cl & 8 & 128 \\
\hline
\end{tabular}

(Fig. 1, line B) under energized conditions was much lower than that in cells of KAM33/pSTV29 (Fig. 1, line A). However, after addition of the $\mathrm{H}^{+}$conductor, carbonylcyanide- $m$-chlorphenylhydrazone (CCCP), intracellular ethidium reached the same levels in both types of cells, demonstrating the energy-dependent nature of ethidium extrusion via VmeAB.

Because two other multidrug efflux transporters from $V$. parahaemolyticus, NorM and VmrA, had proven to be $\mathrm{Na}^{+}$-stimulated $\left(\mathrm{Na}^{+}\right.$-coupled), we also tested the effect

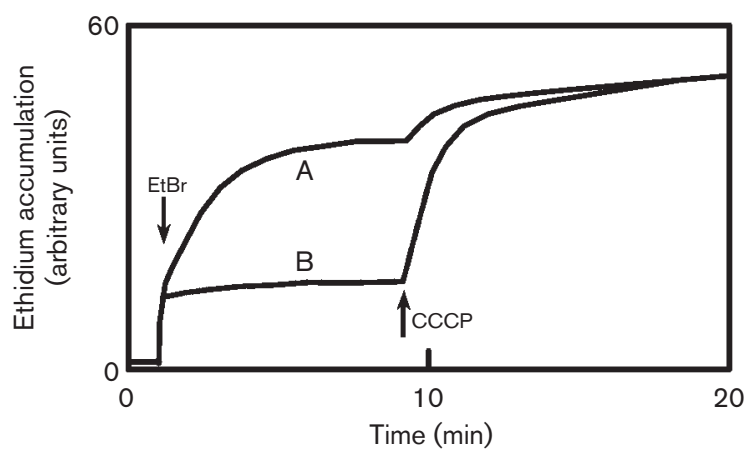

Fig. 1. Ethidium extrusion activity in cells of $E$. coli KAM33/pSTV29 (control, trace A) and KAM33/pRHR229 (carrying vmeAB, trace B). Ethidium bromide $(\mathrm{EtBr})$ was added to cell suspensions at a final concentration of $40 \mu \mathrm{M}$ at the indicated time point. Accumulation of ethidium in the cells was monitored continuously by measuring the fluorescence of ethidium with excitation at $500 \mathrm{~nm}$ and emission at $580 \mathrm{~nm}$. Carbonyl cyanide $m$-chlorophenylhydrazone (CCCP) was added at a final concentration of $100 \mu \mathrm{M}$ to de-energize the cells at the indicated time point. 
Table 2. Complementation of tolC deficiency with $v p o C$ of $V$. parahaemolyticus

\begin{tabular}{|c|c|c|}
\hline \multirow[t]{2}{*}{ Antimicrobial agent } & \multicolumn{2}{|c|}{$\operatorname{MIC}\left(\mu \mathrm{g} \mathrm{ml}^{-1}\right)$} \\
\hline & $\begin{array}{c}\text { TG1 } \Delta \text { tolC/pBR322 } \\
\text { (control) }\end{array}$ & $\begin{array}{c}\text { TG1 } \Delta \text { tolC/pBVT3 } \\
(v p o C)\end{array}$ \\
\hline Erythromycin & 2 & 16 \\
\hline Norfloxacin & 0.015 & 0.06 \\
\hline Novobiocin & 0.25 & 4 \\
\hline Acriflavine & 2 & 8 \\
\hline Ethidium bromide & 2 & 64 \\
\hline Rhodamine 6G & 4 & 16 \\
\hline Sodium deoxycholate & 125 & 4000 \\
\hline SDS & 25 & 400 \\
\hline TPP-Cl & 4 & 64 \\
\hline
\end{tabular}

of $\mathrm{Na}^{+}$on VmeAB-mediated ethidium extrusion. However, addition of $10 \mathrm{mM} \mathrm{NaCl}$ hardly affected ethidium extrusion activity via $\mathrm{VmeAB}$ (data not shown). We also tested whether $\mathrm{Na}^{+}$movement via $\mathrm{VmeAB}$ was observed or not using an $\mathrm{Na}^{+}$-electrode as reported previously (Chen et al., 2002; Morita et al., 2000). However, we observed no detectable $\mathrm{Na}^{+}$movement after the addition of ethidium to the assay mixture (data not shown). Thus, VmeAB is not likely to be a $\mathrm{Na}^{+}$-coupled transporter.

\section{Requirement of TolC for the function of VmeAB}

Although RND-type efflux transporters require an outermembrane component to function in multidrug efflux (Piddock, 2006; Putman et al., 2000), the downstream region of $v m e A B$ in the plasmid pRHR229 contained no gene that might encode an outer-membrane protein; and no such ORF exists, either, in the vicinity of the
VP1091-VP1092 loci of the V. parahaemolyticus genome sequence. In E. coli, RND-type efflux transporters require TolC as their outer-membrane component (Nagakubo et al., 2002; Nishino et al., 2003). We then investigated the possibility that TolC of $E$. coli could be utilized by expressed $V m e A B$, by simply introducing the vmeAB plasmid into the cells of E. coli KAM43 lacking tolC. The resulting cells showed MICs of all of the antimicrobial agents tested to be lower than KAM33 possessing tolC and expressing $v m e A B$ (data not shown). This complementation of $\mathrm{VmeAB}$ by the $E$. coli tolC gene strongly implicates TolC as a functional protein partner of heterologously expressed VmeAB.

In V. parahaemolyticus itself, ORF VP0425 codes for a protein showing high sequence identity $(44 \%)$ and similarity $(88 \%)$ with TolC of E. coli, but VP0425 is located at a distant chromosomal site remote from $v m e A B$. We therefore cloned out VP0425 (designated $v p o C$ ) by PCR and expressed it in E. coli TG1 $\mathrm{t}$ tolC, having the native tolC gene deleted. TolC plays a crucial role in resistance to many antimicrobial agents in E. coli (Fralick, 1996), and TG1 $\operatorname{tol} C$ proved hypersusceptible to those antimicrobial agents (Nagakubo et al., 2002; Nishino et al., 2003). However, when $v p o C$ was expressed in TG1 1 tolC, the transformed strain (TG1 $\Delta$ tolC/pBVT3) showed much higher MICs of the tested agents (Table 2). It became clear that $\mathrm{VpoC}$ was functional when expressed in E. coli cells and that $\mathrm{VpoC}$ could function with some efflux transporters of $E$. coli. Next, in order to investigate the effect of $\mathrm{VpoC}$ on $\mathrm{VmeAB}$ activity, we co-expressed $v p o C$ together with $v m e A B$ in E. coli KAM43, also lacking tolC (see above). Expression of VpoC in KAM43 hardly affected the MICs, possibly because VpoC could function only with AcrAB and/or YdhE in E. coli (Table 3, columns 1 and 2). Additional expression of $\mathrm{VmeAB}$ in KAM43 expressing VpoC showed much higher MICs of the tested antimicrobial

Table 3. VmeAB requires $\mathrm{VpoC}$ for function

\begin{tabular}{|rcccc|}
\hline Antimicrobial agent & \multicolumn{4}{c|}{ MIC $\left(\boldsymbol{\mu g} \mathbf{~ m l}^{-\mathbf{1}}\right)$} \\
\cline { 2 - 5 } Host: & KAM43 & KAM43 & KAM43 & KAM43 \\
Plasmid: & pSTV29/pBR322 & pSTV29/pBVT3 & pRHR229/pBR322 & pRHR229/pBVT3 \\
vpoC: & - & + & - & + \\
vmeAB: & - & - & + & + \\
\hline Erythromycin & 2 & 2 & 2 & 32 \\
Norfloxacin & 0.03 & 0.03 & 0.03 & 0.5 \\
Novobiocin & 0.5 & 1 & 0.5 & 64 \\
Acriflavine & 2 & 2 & 2 & 16 \\
Ethibium bromide & 2 & 2 & 2 & 128 \\
Hoechst 33342 & 0.25 & 0.25 & 0.25 & 32 \\
Rhodamine 6G & 8 & 8 & 8 & 64 \\
Sodium deoxycholate & 125 & 125 & 125 & 4000 \\
SDS & 50 & 50 & 50 & 6400 \\
TPP-Cl & 4 & 4 & 4 & 128 \\
\hline
\end{tabular}


agents, as compared with the control, KAM43 expressing only $\mathrm{VpoC}$ (Table 3 ). Because the substrate specificity for VmeAB expressed in E. coli KAM43 harbouring both pRHR229 and pBVT3 (Table 3) was very similar to that observed in E. coli KAM33 harbouring pRHR229 (Table 1), it seems safe to conclude that $\mathrm{VpoC}$ of $V$. parahaemolyticus does in fact form the third structural element of a tripartite RND-type multidrug efflux transporter based on VmeAB.

\section{Properties of a vmeAB-deficient mutant}

In order to examine the role of $\mathrm{VmeAB}$ in $V$. parahaemolyticus itself, we disrupted the native vmeAB genes, yielding strain TM3. This mutant strain was more sensitive (lower MICs) to Hoechst 33342; and it was slightly more sensitive (slightly lower MICs) to oxacillin, acriflavine and ethidium bromide (Table 4). The MICs of erythromycin, rhodamine 6G, TPP-Cl, SDS and sodium deoxycholate, however, were not changed by the gene disruption, even though these agents proved to be good substrates for VmeAB expressed in E. coli.

We then carried out a survival test in the presence of sodium deoxycholate in order to investigate the bactericidal effect. The survival rate of TM3 was lower than that of AQ3334 (Fig. 2). The survival rate was restored by the introduction of plasmid pRHR229 carrying vmeAB into the TM3 strain. This indicates that one of the functions of $\mathrm{VmeAB}$ might be to protect the organism from the bactericidal effects of sodium deoxycholate.

\section{DISCUSSION}

From its amino acid sequence and its functional characteristics upon expression in E. coli, the $V$. parahaemolyticus multidrug transporter $\mathrm{Vme} A B$ - which we have now isolated and characterized - is clearly that organism's orthologue of AcrAB in E. coli.

Such RND-type efflux transporters, especially AcrAB in $E$. coli, and MexAB in P. aeruginosa, contribute strongly to the

Table 4. Involvement of $\mathrm{VmeAB}$ in intrinsic resistance in $V$. parahaemolyticus

\begin{tabular}{|lcc|}
\hline \multirow{2}{*}{ Antimicrobial agent } & \multicolumn{2}{c|}{ MIC $\left(\boldsymbol{\mu g} \mathbf{~ m l}^{-\mathbf{1}}\right)$} \\
\cline { 2 - 3 } & AQ3334 (parent) & TM3 $(\boldsymbol{\Delta} \boldsymbol{v m e A B})$ \\
\hline Hoechst 33342 & 2 & 0.25 \\
Oxacillin & 128 & 64 \\
Acriflavine & 16 & 8 \\
Ethidium bromide & 64 & 32 \\
Erythromycin & 4 & 4 \\
Norfloxacin & 0.0625 & 0.0625 \\
Rhodamine 6G & 128 & 128 \\
TPP-Cl & 128 & 128 \\
Sodium deoxycholate & 8000 & 8000 \\
\hline
\end{tabular}

intrinsic multidrug resistance of these bacteria ( $\mathrm{Li}$ et al., 1995; Morita et al., 2001; Okusu et al., 1996; Poole et al., 1993). However, the vmeAB-deficient mutant $V$. parahaemolyticus (TM3) showed only modestly lowered MICs of a variety of antimicrobial agents. To explain this phenomenon, there are three possibilities: (1) VmeAB is not expressed in cells of $V$. parahaemolyticus; (2) VmeAB does not function as an efflux transporter in $V$. parahaemolyticus; and (3) the transport activity of other efflux transporter(s) is stronger than that of $V m e A B$, and the contribution of VmeAB is masked. mRNA of $v m e A B$ was detected at a considerable level in $V$. parahaemolyticus by RT-PCR analysis (data not shown), and V. parahaemolyticus TM3 showed reduced survival in the presence of sodium deoxycholate (Fig. 2). Thus, the third possibility seems to be likely. VmeAB is probably not a singular multidrug efflux transporter in $V$. parahaemolyticus, at least under ordinary laboratory conditions. Judging from the genome sequence (Makino et al., 2003), indeed, 11 more putative RND-type efflux transporter genes should be present in cells of $V$. parahaemolyticus, along with nearly 40 other drug efflux transporter genes in different structural classes, which are certainly important for the survival and growth of $V$. parahaemolyticus as a human intestinal parasite.

Interestingly, we did not observe a significant difference between the growth of cells of the parental strain and that of the vmeAB-deleted mutant TM3 in the presence of sodium deoxycholate, but significant differences were observed in their survival rates when exposed to sodium deoxycholate. A possible explanation for this discrepancy is that $\mathrm{VmeAB}$ is involved in the escape from the instantaneous bactericidal effect of sodium deoxycholate, followed by induction of other gene(s) responsible for growth of $V$. parahaemolyticus in the presence of sodium deoxycholate. Since we observed that $v m e A B$ was expressed constitutively, and no induction by deoxycholate was seen (data not shown), it seems possible that expression of some efflux transporters other than $\mathrm{VmeAB}$ was induced, which were involved in the resistance to deoxycholate. Namely, $\mathrm{VmeAB}$ could be involved in the escape from the instantaneous bactericidal effect of deoxycholate, and efflux transporters other than $\mathrm{VmeAB}$ might be involved in the growth in the presence of deoxycholate. In fact, expression of $v e x A B$ and vexCD, which encode RND-type efflux transporters in $V$. cholerae, has been shown to be induced by bile salts (Bina et al., 2006). Our results suggest that other multidrug transporter(s) which is (are) able to extrude deoxycholate is (are) present in V. parahaemolyticus.

AcrB (E. coli) and MexB (P. aeruginosa) are known as $\mathrm{H}^{+}$coupled transporters (Murakami et al., 2002; Ocaktan et al., 1997; Thanassi et al., 1997), and three characteristic charged residues in the transmembrane domains of these two proteins have been suggested to be part of the protontranslocating pathway (Murakami et al., 2002). Since these residues are also conserved in $\mathrm{VmeB}$ of $V$. parahaemolyticus 
(K342, E346, D407, D408, K937), this protein is also likely to be energized by proton coupling, a conclusion that is supported by the finding, reported above, that $\mathrm{Na}^{+}$levels and fluxes are unrelated to VmeAB-mediated transport.

It was shown that $\mathrm{VmeAB}$ could form a functional complex with outer-membrane component TolC of E. coli. Some RND-type efflux transporters have been reported to utilize TolC of E. coli when expressed heterologously in E. coli (Chen et al., 2003; Hansen et al., 2004; Kumar \& Worobec, 2005a; Mine et al., 1999; Srikumar et al., 1998). They are MexCD and MexXY of P. aeruginosa, SdeAB and SdeXY of $S$. marcescens, and OqxAB of E. coli. Disulfide cross-linking data suggested that residues of AcrB interacted directly with TolC (Tamura et al., 2005). However, only G796 was conserved in $\mathrm{VmeB}$ among the five deduced residues. The interaction of $\mathrm{VmeB}$ or AcrB with TolC may require some other residues.

VpoC of V. parahaemolyticus was shown to be a functional orthologue of TolC of E. coli. We showed that introduction of $v p o C$ into E. coli TG1 $\Delta$ tolC conferred elevated resistance to several antimicrobial agents (Table 2). Similar results have been shown with $\mathrm{TolC}_{\mathrm{Vc}}$ of $V$. cholerae (Bina \& Mekalanos, 2001) and HasF of S. marcescens (Kumar \& Worobec, 2005b). On the other hand, the introduction of $v p o C$ into E. coli KAM43 caused an increase in MIC values for only novobiocin (Table 3 ). Since both $a c r A B$ and $y d h E$ were deleted in KAM43, it is likely that VpoC functions with $A$ crAB and/or YdhE, and not with any other efflux transporters in E. coli. As with TolC in E. coli, VpoC might be important in intrinsic resistance to antimicrobial agents in $V$. parahaemolyticus.

According to the published genome sequence, several RND-type efflux transporters seem to be present among the Vibrionaceae, such as V. cholerae, Vibrio vulnificus and Vibrio fischeri (Heidelberg et al., 2000; Makino et al., 2003;

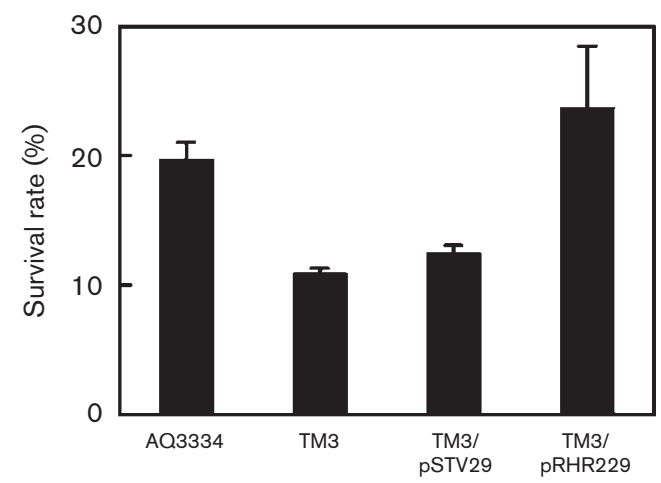

Fig. 2. Effect of sodium deoxycholate on the survival rate of parent and $v m e A B$-deleted $V$. parahaemolyticus. After exposure of $V$. parahaemolyticus cells to $1 \mathrm{mg}$ sodium deoxycholate $\mathrm{ml}^{-1}$, c.f.u. were determined. Error bars represent standard deviations for results from triplicate samples. A representative of two experiments is shown. Similar results were obtained in the other experiment.
Ruby et al., 2005). In fact, two RND-type efflux transporters (VexAB and VexCD) in $V$. cholerae have been characterized (Bina et al., 2006). VmeAB shared high sequence similarity with $\mathrm{Acr} A \mathrm{~B}$ and $\mathrm{Mex} A \mathrm{~B}$, but to a lesser extent with VexAB and VexCD. We could not find such proteins, so-called 'orthologues' among the Vibrionaceae, indiating that $\mathrm{VmeAB}$ is not common among Vibrionaceae. The BLAST search showed that the most similar homologue was SO4692-4693 in Shewanella oneidensis [59\% identity (VmeA versus SO4693), $72 \%$ identity (VmeB versus SO4692)] (Heidelberg et al., 2002).

As described above, 11 more genes for the putative RNDtype efflux transporters are present in the chromosome of $V$. parahaemolyticus. Our present results suggested the contribution of other efflux transporters to resistance against bile acids and antimicrobial agents. We are currently trying to characterize other efflux transporters in $V$. parahaemolyticus.

\section{ACKNOWLEDGEMENTS}

We thank A. Yamaguchi, D. Mazel and B. L. Wanner for providing E. coli strains and plasmids. We also thank C. L. Slayman and M. Varela for critical reading of the manuscript, and S. Shinoda for his helpful discussion. This work was supported by Grants-in-Aid for Scientific Research from the Ministry of Education, Culture, Sports, Science and Technology of Japan.

\section{REFERENCES}

Baumann, P. \& Schubert, R. H. W. (1984). Family II. Vibrionaceae. In Bergey's Manual of Systematic Bacteriology, vol. 1, pp. 516-550. Edited by N. R. Krieg \& J. G. Holt. Baltimore: Williams \& Wilkins.

Berns, K. I. \& Thomas, C. A., Jr (1965). Isolation of high molecular weight DNA from Haemophilus influenzae. J Mol Biol 11, 476-490.

Bina, J. E. \& Mekalanos, J. J. (2001). Vibrio cholerae tolC is required for bile resistance and colonization. Infect Immun 69, 4681-4685.

Bina, J. E., Provenzano, D., Wang, C., Bina, X. R. \& Mekalanos, J. J. (2006). Characterization of the Vibrio cholerae vex $A B$ and vexCD efflux systems. Arch Microbiol 186, 171-181.

Cabanillas-Beltran, H., LLausás-Magaña, E., Romero, R., Espinoza, A., Garcia-Gasca, A., Nishibuchi, M., Ishibashi, M. \& Gomez-Gil, B. (2006). Outbreak of gastroenteritis caused by the pandemic Vibrio parahaemolyticus O3: K6 in Mexico. FEMS Microbiol Lett 265, 76-80.

CDC (2006). Vibrio parahaemolyticus infections associated with consumption of raw shellfish - three states, 2006. MMWR Morb Mortal Wkly Rep 55, 854-856.

Chen, J., Morita, Y., Huda, M. N., Kuroda, T., Mizushima, T. \& Tsuchiya, T. (2002). VmrA, a member of a novel class of $\mathrm{Na}^{+}{ }_{-}$ coupled multidrug efflux pumps from Vibrio parahaemolyticus. J Bacteriol 184, 572-576.

Chen, J., Kuroda, T., Huda, M. N., Mizushima, T. \& Tsuchiya, T. (2003). An RND-type multidrug efflux pump SdeXY from Serratia marcescens. J Antimicrob Chemother 52, 176-179.

Cherepanov, P. P. \& Wackernagel, W. (1995). Gene disruption in Escherichia coli: $\mathrm{Tc}^{\mathrm{R}}$ and $\mathrm{Km}^{\mathrm{R}}$ cassettes with the option of Flpcatalyzed excision of the antibiotic-resistance determinant. Gene 158, 9-14. 
Datsenko, K. A. \& Wanner, B. L. (2000). One-step inactivation of chromosomal genes in Escherichia coli K-12 using PCR products. Proc Natl Acad Sci U S A 97, 6640-6645.

Fralick, J. A. (1996). Evidence that TolC is required for functioning of the Mar/AcrAB efflux pump of Escherichia coli. J Bacteriol 178, 5803-5805.

Hamashima, H., Iwasaki, M. \& Arai, T. (1995). A simple and rapid method for transformation of Vibrio species by electroporation. Methods Mol Biol 47, 155-160.

Hanahan, D., Jessee, J. \& Bloom, F. R. (1991). Plasmid transformation of Escherichia coli and other bacteria. Methods Enzymol 204, 63-113.

Hansen, L. H., Johannesen, E., Burmolle, M., Sorensen, A. H. \& Sorensen, S. J. (2004). Plasmid-encoded multidrug efflux pump conferring resistance to olaquindox in Escherichia coli. Antimicrob Agents Chemother 48, 3332-3337.

Heidelberg, J. F., Eisen, J. A., Nelson, W. C., Clayton, R. A., Gwinn, M. L., Dodson, R. J., Haft, D. H., Hickey, E. K., Peterson, J. D. \& other authors (2000). DNA sequence of both chromosomes of the cholera pathogen Vibrio cholerae. Nature 406, 477-483.

Heidelberg, J. F., Paulsen, I. T., Nelson, K. E., Gaidos, E. J., Nelson, W. C., Read, T. D., Eisen, J. A., Seshadri, R., Ward, N. \& other authors (2002). Genome sequence of the dissimilatory metal ion-reducing bacterium Shewanella oneidensis. Nat Biotechnol 20, 1118-1123.

Henikoff, S. (1984). Unidirectional digestion with exonuclease III creates targeted breakpoints for DNA sequencing. Gene 28, 351-359.

Herz, K., Vimont, S., Padan, E. \& Berche, P. (2003). Roles of NhaA, $\mathrm{NhaB}$, and $\mathrm{NhaD} \mathrm{Na}{ }^{+} / \mathrm{H}^{+}$antiporters in survival of Vibrio cholerae in a saline environment. J Bacteriol 185, 1236-1244.

Huda, M. N., Chen, J., Morita, Y., Kuroda, T., Mizushima, T. \& Tsuchiya, T. (2003). Gene cloning and characterization of VcrM, a $\mathrm{Na}^{+}$-coupled multidrug efflux pump, from Vibrio cholerae non-O1. Microbiol Immunol 47, 419-427.

Japanese Society of Chemotherapy (1990). Microbroth dilution methods for determination of minimum inhibitory concentrations. Chemotherapy 38, 102-105.

Klein, J. R., Henrich, B. \& Plapp, R. (1991). Molecular analysis and nucleotide sequence of the envCD operon of Escherichia coli. Mol Gen Genet 230, 230-240.

Kumar, A. \& Worobec, E. A. (2005a). Cloning, sequencing, and characterization of the SdeAB multidrug efflux pump of Serratia marcescens. Antimicrob Agents Chemother 49, 1495-1501.

Kumar, A. \& Worobec, E. A. (2005b). HasF, a TolC-homolog of Serratia marcescens, is involved in energy-dependent efflux. Can J Microbiol 51, 497-500.

Kumazawa, N. H. \& Kato, E. (1985). Survival of Kanagawa-positive strains of Vibrio parahaemolyticus in a brackish-water area. J Hyg (Lond) 95, 299-307.

Kumazawa, N. H., Fukuma, N. \& Komoda, Y. (1991). Attachment of Vibrio parahaemolyticus strains to estuarine algae. J Vet Med Sci 53, 201-205.

Kuroda, T., Mizushima, T. \& Tsuchiya, T. (2005). Physiological roles of three $\mathrm{Na}^{+} / \mathrm{H}^{+}$antiporters in the halophilic bacterium Vibrio parahaemolyticus. Microbiol Immunol 49, 711-719.

Li, X. Z., Nikaido, H. \& Poole, K. (1995). Role of mexA-mexB-oprM in antibiotic efflux in Pseudomonas aeruginosa. Antimicrob Agents Chemother 39, 1948-1953.

Lozano-Leon, A., Torres, J., Osorio, C. R. \& Martinez-Urtaza, J. (2003). Identification of tdh-positive Vibrio parahaemolyticus from an outbreak associated with raw oyster consumption in Spain. FEMS Microbiol Lett 226, 281-284.
Ma, D., Cook, D. N., Alberti, M., Pon, N. G., Nikaido, H. \& Hearst, J. E. (1993). Molecular cloning and characterization of acrA and acrE genes of Escherichia coli. J Bacteriol 175, 6299-6313.

Ma, D., Cook, D. N., Hearst, J. E. \& Nikaido, H. (1994). Efflux pumps and drug resistance in gram-negative bacteria. Trends Microbiol 2, 489-493.

Makino, K., Oshima, K., Kurokawa, K., Yokoyama, K., Uda, T., Tagomori, K., lijima, Y., Najima, M., Nakano, M. \& other authors (2003). Genome sequence of Vibrio parahaemolyticus: a pathogenic mechanism distinct from that of $V$. cholerae. Lancet 361, 743-749.

Masaoka, Y., Ueno, Y., Morita, Y., Kuroda, T., Mizushima, T. \& Tsuchiya, T. (2000). A two-component multidrug efflux pump, EbrAB, in Bacillus subtilis. J Bacteriol 182, 2307-2310.

McLaughlin, J. B., DePaola, A., Bopp, C. A., Martinek, K. A., Napolilli, N. P., Allison, C. G., Murray, S. L., Thompson, E. C., Bird, M. M. \& Middaugh, J. P. (2005). Outbreak of Vibrio parahaemolyticus gastroenteritis associated with Alaskan oysters. N Engl J Med 353, 1463-1470.

Mine, T., Morita, Y., Kataoka, A., Mizushima, T. \& Tsuchiya, T. (1999). Expression in Escherichia coli of a new multidrug efflux pump, MexXY, from Pseudomonas aeruginosa. Antimicrob Agents Chemother 43, 415-417.

Morita, Y., Kodama, K., Shiota, S., Mine, T., Kataoka, A., Mizushima, T. \& Tsuchiya, T. (1998). NorM, a putative multidrug efflux protein, of Vibrio parahaemolyticus and its homolog in Escherichia coli. Antimicrob Agents Chemother 42, 1778-1782.

Morita, Y., Kataoka, A., Shiota, S., Mizushima, T. \& Tsuchiya, T. (2000). NorM of Vibrio parahaemolyticus is an $\mathrm{Na}^{+}$-driven multidrug efflux pump. J Bacteriol 182, 6694-6697.

Morita, Y., Komori, Y., Mima, T., Kuroda, T., Mizushima, T. \& Tsuchiya, T. (2001). Construction of a series of mutants lacking all of the four major mex operons for multidrug efflux pumps or possessing each one of the operons from Pseudomonas aeruginosa PAO1: MexCD-OprJ is an inducible pump. FEMS Microbiol Lett 202, 139-143.

Murakami, S., Nakashima, R., Yamashita, E. \& Yamaguchi, A. (2002). Crystal structure of bacterial multidrug efflux transporter AcrB. Nature 419, 587-593.

Nagakubo, S., Nishino, K., Hirata, T. \& Yamaguchi, A. (2002). The putative response regulator BaeR stimulates multidrug resistance of Escherichia coli via a novel multidrug exporter system, MdtABC. J Bacteriol 184, 4161-4167.

Nishino, K. \& Yamaguchi, A. (2001). Analysis of a complete library of putative drug transporter genes in Escherichia coli. J Bacteriol 183, 5803-5812.

Nishino, K., Yamada, J., Hirakawa, H., Hirata, T. \& Yamaguchi, A. (2003). Roles of TolC-dependent multidrug transporters of Escherichia coli in resistance to $\beta$-lactams. Antimicrob Agents Chemother 47, 3030-3033.

Obata, H., Kai, A. \& Morozumi, S. (2001). The trends of Vibrio parahaemolyticus foodborne outbreaks in Tokyo: 1989-2000. Kansenshogaku Zasshi 75, 485-489.

Ocaktan, A., Yoneyama, H. \& Nakae, T. (1997). Use of fluorescence probes to monitor function of the subunit proteins of the MexAMexB-OprM drug extrusion machinery in Pseudomonas aeruginosa. J Biol Chem 272, 21964-21969.

Okusu, H., Ma, D. \& Nikaido, H. (1996). AcrAB efflux pump plays a major role in the antibiotic resistance phenotype of Escherichia coli multiple-antibiotic-resistance (Mar) mutants. J Bacteriol 178, 306-308.

Piddock, L. J. (2006). Multidrug-resistance efflux pumps - not just for resistance. Nat Rev Microbiol 4, 629-636. 
Poole, K., Krebes, K., McNally, C. \& Neshat, S. (1993). Multiple antibiotic resistance in Pseudomonas aeruginosa: evidence for involvement of an efflux operon. J Bacteriol 175, 7363-7372.

Provenzano, D., Schuhmacher, D. A., Barker, J. L. \& Klose, K. E. (2000). The virulence regulatory protein ToxR mediates enhanced bile resistance in Vibrio cholerae and other pathogenic Vibrio species. Infect Immun 68, 1491-1497.

Putman, M., van Veen, H. W. \& Konings, W. N. (2000). Molecular properties of bacterial multidrug transporters. Microbiol Mol Biol Rev 64, 672-693.

Ruby, E. G., Urbanowski, M., Campbell, J., Dunn, A., Faini, M., Gunsalus, R., Lostroh, P., Lupp, C., McCann, J. \& other authors (2005). Complete genome sequence of Vibrio fischeri: a symbiotic bacterium with pathogenic congeners. Proc Natl Acad Sci U S A 102, 3004-3009.

Sekiya, H., Mima, T., Morita, Y., Kuroda, T., Mizushima, T. \& Tsuchiya, T. (2003). Functional cloning and characterization of a multidrug efflux pump, mexHI-opmD, from a Pseudomonas aeruginosa mutant. Antimicrob Agents Chemother 47, 2990-2992.

Sen, B., Dutta, B., Chatterjee, S., Bhattacharya, M. K., Nandy, R. K., Mukhopadhyay, A. K., Gangopadhyay, D. N., Bhattacharya, S. K. \& Ramamurthy, T. (2007). The first outbreak of acute diarrhea due to a pandemic strain of Vibrio parahaemolyticus O3: K6 in Kolkata, India. Int J Infect Dis 11, 185-187.

Srikumar, R., Kon, T., Gotoh, N. \& Poole, K. (1998). Expression of Pseudomonas aeruginosa multidrug efflux pumps MexA-MexB-OprM and MexC-MexD-OprJ in a multidrug-sensitive Escherichia coli strain. Antimicrob Agents Chemother 42, 65-71.

Su, H. P., Chiu, S. I., Tsai, J. L., Lee, C. L. \& Pan, T. M. (2005a). Bacterial food-borne illness outbreaks in northern Taiwan, 19952001. J Infect Chemother 11, 146-151.
Su, X. Z., Chen, J., Mizushima, T., Kuroda, T. \& Tsuchiya, T. (2005b). AbeM, an $\mathrm{H}^{+}$-coupled Acinetobacter baumannii multidrug efflux pump belonging to the MATE family of transporters. Antimicrob Agents Chemother 49, 4362-4364.

Tamura, N., Murakami, S., Oyama, Y., Ishiguro, M. \& Yamaguchi, A. (2005). Direct interaction of multidrug efflux transporter AcrB and outer membrane channel TolC detected via site-directed disulfide cross-linking. Biochemistry 44, 11115-11121.

Thanassi, D. G., Cheng, L. W. \& Nikaido, H. (1997). Active efflux of bile salts by Escherichia coli. J Bacteriol 179, 2512-2518.

Tseng, T. T., Gratwick, K. S., Kollman, J., Park, D., Nies, D. H., Goffeau, A. \& Saier, M. H., Jr (1999). The RND permease superfamily: an ancient, ubiquitous and diverse family that includes human disease and development proteins. J Mol Microbiol Biotechnol 1, $107-125$.

WHO (1999). Vibrio parahaemolyticus, Japan, 1996-1998. Wkly Epidemiol Rec 74, 361-363.

Xu, X. J., Su, X. Z., Morita, Y., Kuroda, T., Mizushima, T. \& Tsuchiya, T. (2003). Molecular cloning and characterization of the HmrM multidrug efflux pump from Haemophilus influenzae Rd. Microbiol Immunol 47, 937-943.

Yamamoto, T. \& Yokota, T. (1989). Adherence targets of Vibrio parahaemolyticus in human small intestines. Infect Immun 57, 2410-2419.

Yamazaki, M., Inuzuka, K., Matsumoto, M., Miwa, Y., Hiramatsu, R., Matsui, H., Sakae, K., Suzuki, Y. \& Miyazaki, Y. (2003). Epidemiological study of outbreaks and sporadic cases due to Vibrio parahaemolyticus - serotype O3:K6 in Aichi Prefecture, Japan, during 1988 and 2001. Kansenshogaku Zasshi 77, 1015-1023.

Edited by: T. Abee 